\title{
MÉTODO MÃE CANGURU PARA RECÉM-NASCIDOS DE BAIXO PESO: REVISÃO DA LITERATURA
}

\section{Carolina Correia Bilotti}

Mestranda no Programa de Pós-graduação em Promoção da Saúde pelo Centro Universitário de Maringá (UNICESUMAR), Maringá (PR), Brasil; Bolsista CAPES

E-mail: carolinac_bilotti@hotmail.com

\section{Eliane da Silva Gomes}

Mestranda no Programa de Pós-graduação em Promoção da Saúde pelo Centro Universitário de Maringá (UNICESUMAR), Maringá (PR), Brasil.

\section{Adriane Behring Bianchi}

Mestranda no Programa de Pós-graduação em Promoção da Saúde pelo Centro Universitário de Maringá (UNICESUMAR), Maringá (PR), Brasil; Bolsista CAPES

\section{Ludmila Lopes Maciel Bolsoni}

Mestre no Programa de Pós-graduação em Promoção da Saúde pelo Centro Universitário de Maringá (UNICESUMAR), Docente dos cursos Bacharel em Enfermagem e Medicina do Centro Universitário de Maringá (UNICESUMAR) Maringá (PR), Brasil.

\section{Sandra Mara Aparecida Santos}

Mestre no Programa de Pós-graduação em Promoção da Saúde pelo Centro Universitário de Maringá (UNICESUMAR); Discente de Enfermagem Obstetrícia e Neonatal pela Universidade Estadual do Paraná (Unespar) Londrina (PR), Brasil.

\section{Marcelo Picinin Bernuci}

Docente Permanente do Programa de Pós-graduação em Promoção da Saúde e Tecnologias Limpas e do Curso de Medicina no Centro Universitário de Maringá (UNICESUMAR); Pesquisador no Instituto Cesumar de Ciência, Tecnologia e Inovação (ICETI), Maringá (PR), Brasil
RESUMO: Este estudo teve como objetivo comparar o Método Mãe Canguru com o cuidado convencional dos recém-nascidos de baixo peso em uma Revisão Sistematizada PRISMA. De maio a junho de 2015, pesquisou-se na literatura artigos que empregavam o Método Mãe Canguru, para comparar com outros métodos de cuidado para recém-nascidos de baixo peso. Encontrou-se 246 artigos publicados na Cochrane Library. Os artigos passaram por uma sequência de triagem resultando em 13 artigos incluídos no presente estudo. Os resultados da aplicação do Método Mãe Canguru demonstram que não há prejuízo para o recém-nascido de baixo peso, e os resultados foram similares ou com vantagens para o Método Mãe Canguru. O presente estudo sugere que o Método Mãe Canguru auxilia positivamente no desenvolvimento do recém-nascido de baixo peso, com maior ganho de peso, auxiliando a formação do vínculo afetivo mãe-filho, aumentando adesão ao aleitamento materno, auxiliando na redução de custos hospitalares e do período de internação.

PALAVRAS-CHAVE: Unidades de terapia intensiva neonatal; Ganho de peso; Humanização da assistência; Método canguru.

\section{MOTHER KANGAROO METHOD FOR LOW-WEIGHT RECENTLY-BORN BABIES: A REVIEW OF THE LITERATURE}

\begin{abstract}
Current analysis compares Mother Kangaroo Method and conventional care to low-weight newly-born children in a Prisma systemized revision. Articles featuring the Mother Kangaroo Method were researched between May and June 2015 and compared with other care methods for low weight recently born children. Two hundred and forty-six published articles could be found in the Cochrane Library. Thirteen articles were selected and were included in current analysis. Results for the application of the Mother Kangaroo Method showed that no inconveniency occurred to low-weight children and results were similar to or better for the Mother Kangaroo Method. Current investigation suggests that Mother Kangaroo Method positively helps the development of low-weight newly-born children through a greater weight gain, formation of an affection bond between mother and child, increase in the adhesion to breast feeding, reduction in hospital costs and hospitalization period.
\end{abstract}

KEY WORDS: Intensive care units, neonatal; Weight gain; Humanization of assistance; Kangaroo-Mother Care Method. 


\section{INTRODUÇão}

O Método Mãe-Canguru (MMC) é o tipo de assistência neonatal voltada para $o$ atendimento do recém-nascido que implica em colocar o bebê em contato pele a pele com a sua mãe ou substituto, com início precocemente, continuando depois da alta hospitalar (BERA et al., 2014). Diante do seu contexto histórico, o MMC foi desenvolvido na cidade de Bogotá, na Colômbia, entre 1979 e 1978, pelos médicos Edgar Rey Sanabria e Hector Martinez (WHITELAW et al., 1988 NAGAI et al., 2010), com intuito de melhorar os cuidados prestados ao recém-nascido prematuro com foco na redução do tempo de permanência hospitalar. No Brasil, o MMC tem ocupado seu espaço desde 1992 (BRASIL, 2011), sendo o Hospital Guilherme Álvaro, em Santos e o Instituto Materno-Infantil de Pernambuco, os pioneiros na introdução dessa técnica de cuidado no Brasil, iniciando de forma sistematizada esse novo protocolo de cuidado aos recém-nascidos (MACHADO et al., 2012).

O MMC fundamenta-se no processo de desenvolvimento contínuo do bebê, com introdução de algumas possibilidades de entendimento da assistência neonatal em um contexto mais amplo. Busca-se com esse método o resgate dos conhecimentos fisiológicos, psicológicos e neurológicos do ser humano que consideram o indivíduo por completo e não apenas um corpo dividido em sistemas isolados (BRASIL, 2002). A prática do MMC objetiva-se em atingir uma considerável redução da morbimortalidade neonatal, valorização da atenção ao recém-nascido de baixo peso (RNBP), promoção do aleitamento materno exclusivo, acompanhamento do crescimento e desenvolvimento da criança, promoção das imunizações, prevenção e controle de infecções perinatais (CARDOSO et al., 2006).

Observa-se em algumas regiões do Brasil um aumento do número de nascimentos de recém-nascidos prematuros e de baixo peso, além das ainda altas taxas de mortalidade neonatal, com constante elevação de custos hospitalares e elevado índice de sequelas no crescimento e desenvolvimento neonatal e infantil, que configuram algumas das grandes preocupações da classe médica atual (ALMEIDA et al., 2011; VICTORA et al., 2011; SPEHAR, 2013; WHO, 2012). Considerando que esses
RNBP necessitarão de cuidados específicos, destaca-se ainda que o tratamento hospitalar convencional, além de ser um atendimento de custo elevado, distancia a mãe de seu bebê, podendo aumentar o índice de infecção. Com vista a isso torna-se necessária a comprovação da eficácia de métodos de cuidados neonatal alternativos. $\mathrm{O}$ presente estudo busca revisar os artigos que descrevam a aplicação do MMC como método de cuidado para os RNPB, identificando os benefícios no processo de ganho de peso, no desenvolvimento e benefícios adicionais.

\section{METODOLOGIA}

A Revisão Sistemática da literatura desse estudo teve como pergunta norteadora: Quais os benefícios do MMC no processo de desenvolvimento e do ganho de peso dos RNBP.

Foram pesquisados na literatura durante os meses de maio e junho de 2015 artigos que empregavam o MMC, comparando com outros métodos de cuidado para RNBP, utilizando-se as recomendações do protocolo de apresentação de Revisões Sistemáticas PRISMA (PRISMA, 2015). A pesquisa concentrou-se na base de dados Cochrane Library, utilizando os seguintes descritores: Kangaroo mother care e Skin-to-skin contact after birth.

Foram incluídos estudos originais, do tipo ensaio clínico, independente do tempo de seguimento, que descrevessem resultados relativos à adoção do MMC, e que comparassem o MMC com o método tradicional de cuidado, avaliando o desenvolvimento e o ganho de peso de RNBP. Os demais critérios de inclusão foram: artigos publicados na base de dados Cochrane Library, com foco em RNBP, e publicados nos idiomas inglês, espanhol ou português. Foram excluídos os estudos duplicados, aqueles que não estavam disponíveis publicamente na íntegra e revisões de literatura sobre o tema.

\section{RESULTADOS}

O método de busca adotado resultou em um total de 246 artigos publicados na Cochrane Library. Os artigos passaram por uma sequência de triagem: na 
primeira etapa, após análise dos títulos dos estudos, 207 trabalhos foram excluídos por não enquadrarem-se nos critérios de inclusão. Na segunda etapa os resumos foram analisados levando a exclusão de mais 16 artigos por não estarem disponíveis publicamente na íntegra. A última etapa consistiu em uma leitura completa dos manuscritos resultando na eliminação de mais 10 artigos por se tratarem de estudos duplicados. Ao final, 13 artigos foram incluídos no presente estudo (Figura 1).

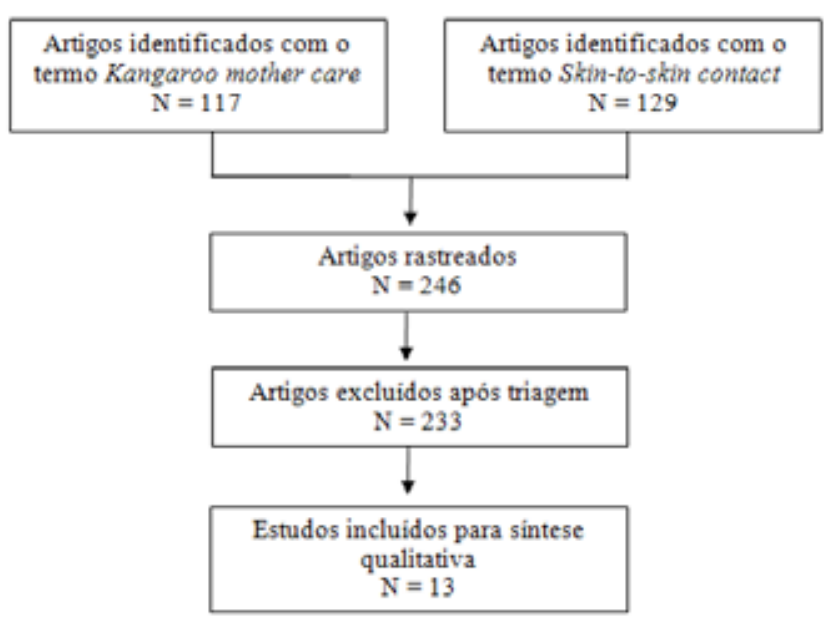

Figura 1. Descrição da seleção dos estudos incluídos na revisão sistemática sobre os benefícios do método canguru no processo de ganho de peso e no desenvolvimento dos recém-nascidos de baixo peso.

Inicialmente analisou-se quais foram os países onde os estudos foram originados. A figura 2 apresenta um panorama desses países, destacando que $86 \%$ deles foram conduzidos em países subdesenvolvidos e apenas $16 \%$ realizados em países desenvolvidos.

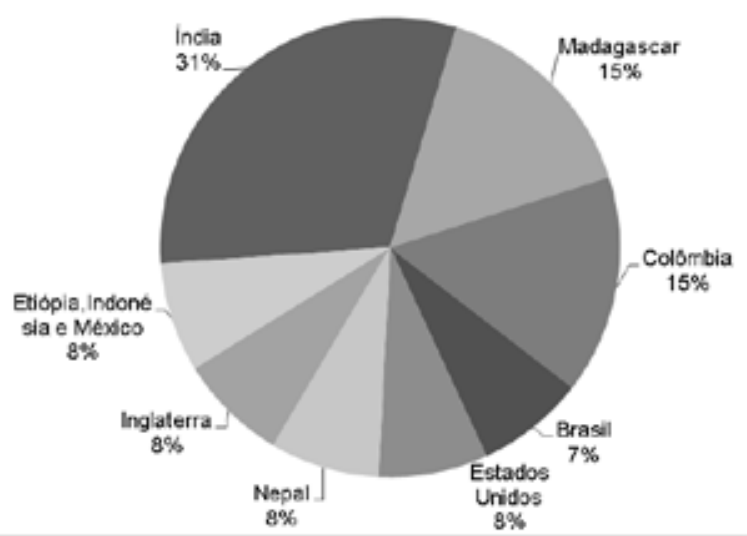

Figura 2. Relação dos países onde os estudos foram conduzidos. Porcentagem do número de estudos realizados adotando o MMC no cuidado ao RNBP por país.
Para avaliar as vantagens ou desvantagens do MMC, todos os estudos usaram grupos de comparação compostos por recém-nascidos com características semelhantes. Em alguns locais onde os estudos foram realizados, por ser considerado o tratamento padrão para RNBP, em respeito às questões éticas, pelo desejo das mães ou por apresentarem maior fragilidade, diversos RNBP foram alocados preferencialmente no grupo MMC. A figura 3 apresenta como foram conduzidas as distribuições dos grupos para realização dos estudos, sendo que em $69 \%$ dos estudos foram utilizados apenas um grupo controle onde os RNBP recebiam os cuidados convencionais em incubadora comparando os resultados obtidos com os recém-nascidos que recebiam os cuidados do MMC.

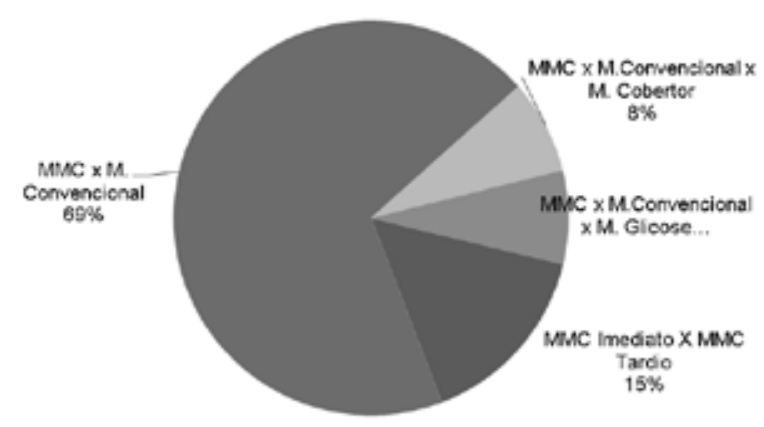

Figura 3. Composição dos grupos de comparação dos estudos revisados. Modo como ocorreu à divisão dos RNPB para a realização das intervenções

O aleitamento materno exclusivo como forma de nutrição foi escolha de $38 \%$ das mães dos estudos analisados, o leite materno com suplementação se necessária ocorreu em $31 \%$ das mães dos estudos, no entanto em $31 \%$ dos estudos não foi informado o tipo de nutrição adotada como demonstrado na figura 4 . Destaca-se que em todos os estudos as mães receberam orientações sobre a maneira de conduzir o MMC e a forma como deveriam amamentar seus bebês, salientando-se que os grupos submetidos ao MMC apresentaram maior incidência de amamentação exclusiva até os seis meses de idade (CHARPAK et al., 1997; CATTANEO et al., 1998; ALI et al., 2009; NAGAI et al., 2011). Visto que, quanto mais cedo o MMC for iniciado, maior será a taxa de adesão à amamentação (NAGAI et al., 2011), uma vez que o método é tido como um facilitador do processo de amamentação. 


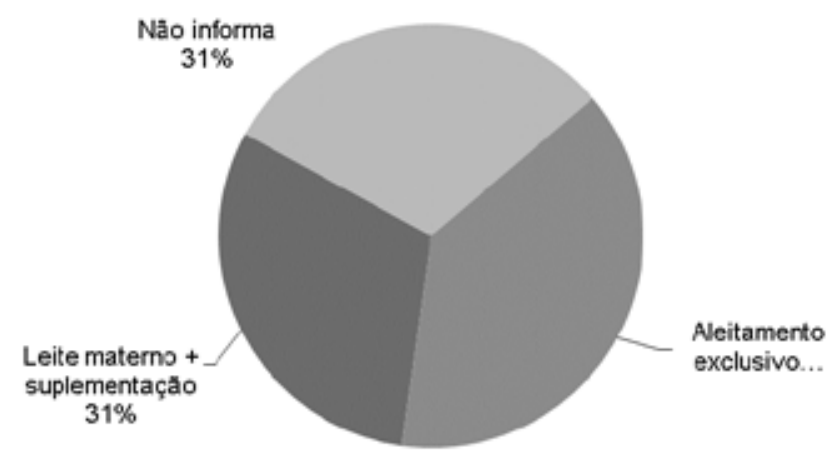

Figura 4. Tipo de nutrição utilizada nos estudos. Relação da escolha do tipo de nutrição dos RNBP que compunham as amostras dos estudos revisados.
O tempo de acompanhamento dos recémnascidos nos estudos variou de um dia a 42 meses, sendo que em $46 \%$ dos estudos analisados prevaleceu um acompanhamento de 12 meses. Em relação ao tamanho da amostra, foi detectada uma disparidade, variando de 71 a 777 recém-nascidos por estudo, no entanto esses valores se alteraram ao longo dos experimentos, uma vez que muitos estudos tiveram sua amostra reduzida devido ao óbito dos RNBP, ou por algumas mães não conseguirem implementar o MMC e devido mudança de endereço das famílias (CHARPAK et al., 1997; CATTANEO et al., 1998; SUMAN RAO; UDANI; NANAVATI, 2008; NAGAI, 2010; NAGAI, 2011; BERA, 2014).

Quadro 1. Síntese de resultados dos artigos analisados. Tamanho da amostra e duração dos estudos

(continua)

\begin{tabular}{|c|c|c|c|}
\hline Autor e ano & N. amostral & $\begin{array}{l}\text { Tempo de } \\
\text { acompanhamento }\end{array}$ & Resultado obtido \\
\hline Bera et al. 2014 & 500 & 42 meses & $\begin{array}{l}\text { Bebês do grupo MMC apresentaram maior crescimento e melhor } \\
\text { desenvolvimento motor. }\end{array}$ \\
\hline $\begin{array}{c}\text { Nagai et al. }{ }^{22} \\
2011\end{array}$ & 73 & 12 meses & $\begin{array}{l}\text { Não houve diferença na mortalidade e reinternação entre os grupos. Relação } \\
\text { peso-altura e peso-idade apresentou-se maior no grupo MMC imediato. }\end{array}$ \\
\hline $\begin{array}{l}\text { Nagai et al. } \\
2010\end{array}$ & 73 & 28 dias & $\begin{array}{l}\text { A aplicação do MMC imediato não interferiu na mortalidade. Embora as } \\
\text { diferenças não sejam significativas, houve um favorecimento da técnica MMC } \\
\text { imediato. }\end{array}$ \\
\hline $\begin{array}{l}\text { Freire et al. } \\
\quad 2008\end{array}$ & 95 & 1 dia & $\begin{array}{l}\text { A aplicação do MMC resultou em um efeito analgésico melhor que os demais } \\
\text { métodos. Com ritmo cardíaco menor que nos demais métodos, saturação parcial } \\
\text { de } \mathrm{O} 2 \text { menor que no método tradicional e indicadores comportamentais igual } \\
\text { ao método glicose. }\end{array}$ \\
\hline Neu et al. 2014 & 87 & 8 semanas & Os lactantes dos diferentes grupos tiveram resultados semelhantes. \\
\hline $\begin{array}{c}\text { Tessier et al. } \\
2003\end{array}$ & 431 & 12 meses & $\begin{array}{l}\text { O MMC proporcionou um ambiente melhor comparado ao M. tradicional para } \\
\text { os prematuros, tendo efeito positivo na audição e fala. }\end{array}$ \\
\hline Ali et al. 2009 & 114 & 12 meses & $\begin{array}{l}\text { Maior ganho de peso no grupo MMC. Infecções graves ocorreram com maior } \\
\text { frequência no grupo controle e amamentação exclusiva mais prevalente no } \\
\text { grupo MMC. }\end{array}$ \\
\hline $\begin{array}{l}\text { Suman Rao et } \\
\text { al. } 2008\end{array}$ & 206 & 9 meses & $\begin{array}{l}\text { Peso, comprimento e circunferência da cabeça foram significativamente maiores } \\
\text { no grupo MMC ao fim do estudo. As morbidades foram significativamente mais } \\
\text { prevalentes no grupo controle. }\end{array}$ \\
\hline
\end{tabular}


(conclusão)

\begin{tabular}{|c|c|c|c|}
\hline Autor e ano & N. amostral & $\begin{array}{c}\text { Tempo de } \\
\text { acompanhamento }\end{array}$ & Resultado obtido \\
\hline $\begin{array}{c}\text { Cattaneo et al. } \\
1998\end{array}$ & 100 & 12 meses & $\begin{array}{l}\text { Grupo MMC alcançou o peso de alta em menor tempo, teve alta hospitalar } \\
\text { mais rapidamente, resultando em um custo de internação } 50 \% \text { menor quando } \\
\text { comparado ao método tradicional. }\end{array}$ \\
\hline $\begin{array}{c}\text { Ghavane et al. } \\
2012\end{array}$ & 140 & 22 meses & $\begin{array}{l}\text { Não houve diferença no tempo e no ganho de peso e a duração da internação foi } \\
\text { semelhante. No entanto, o estudo aponta que o MMC pode ser uma alternativa } \\
\text { relatando uma economia no custo da internação dos bebês MMC de 500 dólares } \\
\text { para cada paciente. }\end{array}$ \\
\hline $\begin{array}{c}\text { Charpak et al. } \\
1997\end{array}$ & 777 & 12 meses & $\begin{array}{l}\text { O estudo apontou que não há redução no crescimento físico dos bebês } \\
\text { submetidos ao MMC, além de ter encontrado uma economia de 1,1 dias de } \\
\text { período de internação dos bebês MMC. }\end{array}$ \\
\hline $\begin{array}{c}\text { Acharya et al. } \\
2014\end{array}$ & 126 & 12 meses & $\begin{array}{l}\text { Grupo MMC apresentou maior ganho de peso. Os quadros de hipotermia } \\
\text { foram significativamente menores no grupo MMC. No entanto a internação foi } \\
\text { significativamente maior no grupo MMC. }\end{array}$ \\
\hline $\begin{array}{c}\text { Whitelaw et al. } \\
1988\end{array}$ & 71 & 18 meses & $\begin{array}{l}\text { Grupo MMC apresentou resultados significativamente melhores em relação a } \\
\text { duração da lactação e na redução do choro. Embora não influencie na melhora } \\
\text { da confiança das mães em relação aos cuidados com o bebê. }\end{array}$ \\
\hline
\end{tabular}

Fonte: Dados da pesquisa

O Quadro 1 descreve os resultados obtidos nas intervenções realizadas nos 13 estudos selecionados para essa revisão, destacando como benefícios do MMC o ganho de peso dos recém-nascidos e a economia em relação ao custo de hospitalização nos grupos submetidos ao MMC. Os resultados da aplicação do MMC demonstram diversos efeitos positivos para o cuidado dos RNBP, sendo: maior estímulo ao aleitamento materno com maior adesão, pois permite maior frequência e duração (WHITELAW et al., 1988; CATTANEO et al., 1998; SUMAN RAO; UDANI; NANAVATI, 2008; ALI et al., 2009; NAGAI et al., 2011), mais rápido ganho de peso neonatal (CATTANEO et al., 1998; SUMAN RAO; UDANI; NANAVATI, 2008; ALI et al., 2009; ACHARYA et al., 2014) e com isso tempo de internação menor (CATTANEO et al., 1998; ALI et al., 2009), crescimento em comprimento e perímetro cefálico mais rápido (SUMAN RAO; UDANI; NANAVATI, 2008), redução do choro do bebê após a alta hospitalar (WHITELAW et al.,1988), redução dos riscos de sepse durante a internação (SUMAN RAO; UDANI; NANAVATI, 2008; ALI et al., 2009) , minimização do estresse por dor (FREIRE; GARCIA; LAMY, 2008), além de favorecer um melhor desenvolvimento neuropsicomotor (TESSIER et al., 2003; BERA et al., 2014), melhor estimulo sensorial, e ser mais econômico (GHAVANE et al., 2012).

Não houve diferenças estatisticamente significativas na mortalidade dos RNBP nos primeiros 28 dias de vida, nem na duração do internamento, nem na morbidade na comparação de MMC iniciado precocemente, antes de $24 \mathrm{~h}$, ou após $24 \mathrm{~h}$, mas a perda de peso inicial dos RNBP foi significativamente menor foi quando o MMC foi iniciado antes de $24 \mathrm{~h}$ de vida (NAGAI et al., 2010).

\section{DISCUSSÃO}

Os diversos países encontrados desenvolvendo trabalhos com o MMC demonstram que a divulgação do método promovida pelo Fundo das Nações Unidas para a Infância (UNICEF), baseada nos casos de sucessos das experiências da Colômbia, surtiu efeito, uma vez que países com diferentes características adotaram o método (Figura 2). O Brasil ao adotar o método optou por utilizar apenas o termo Método Canguru por considerar essa uma experiência na qual os outros familiares podem ter participação ativa também, além de ser o primeiro país a tornar o método uma política pública e 
padronizar seus procedimentos (CARDOSO et al., 2006). Embora esta seja uma política pública implantada no país, o presente estudo encontrou apenas um estudo experimental (FREIRE; GARCIA; LAMY, 2008) que avaliou os benefícios do cuidado MMC comparando a outros métodos de cuidado. Salienta-se assim a necessidade de mais pesquisas práticas que avaliem os reais benefícios para RNBP, familiares, equipe profissional e o benefício financeiro do método.

Vale ressaltar que a forma de uso e os objetivos ao implantar o MMC divergem entre os países, estando relacionado diretamente ao seu grau de desenvolvimento, levando ainda em consideração os valores culturais e as crenças da população em questão (ROBINSON; SCHMIEGE, 2013; ACHARYA et al., 2014; BERA et al., 2014). Observa-se uma tendência dos países que dispõem de número insuficiente de incubadoras e de recursos humanos a indicar o cuidado MMC, pois é mais econômico (GHAVANE et al., 2012; BERA et al., 2014). Nesse sentido, o ato de carregar o RNBP contra o tórax se expande pelo mundo levando a uma substituição da "máquina" pelo "humano", fazendo com que a crônica dificuldade de se obter recursos adequados para a saúde acene para essa metodologia simples, vantajosa e de baixo custo (BRASIL, 2011).

A figura 3 apresenta as proporções dos grupos de estudo distribuídas entre grupos MMC comparados com grupos controle, e corrobora com dados da literatura que demonstra ser frequente a concentração de estudos relacionados ao MMC que avaliam a evolução dos RNPB comparando com os cuidados dos métodos convencionais (MOTA, 2005). Trabalhos na área de cuidados humanizados a recém-nascidos sugerem que a utilização do MMC em RNBP é uma alternativa ao cuidado neonatal convencional, principalmente, quando se considera situações de recursos limitados (CHARPAK et al., 1997; GHAVANE et al., 2012).

Mesmo após a alta dos RNBP em 46\% dos estudos analisados as mães foram encorajadas a continuar com o MMC em casa (WHITELAW et al., 1988; GHAVANE et al., 2012; BERAet al., 2014). O MMC possui como característica fundamental sua acessibilidade, uma vez que o método pode ser realizado em qualquer situação ou contexto, não necessitando de equipamentos ou tecnologias especiais
(BERA et al., 2014). Ressalta-se a viabilidade do MMC a ser continuado pelas famílias após a alta hospitalar, desde que o MMC seja iniciado no hospital com estreita supervisão e orientação (SUMAN RAO; UDANI; NANAVATI, 2008). Contudo, deve-se destacar que a aplicação dos cuidados MMC não visa substituir as UTI (unidades de tratamento intensivo) neonatais nem mesmo reduzir os custos com recursos humanos e tecnológicos, mas sim adicionar uma nova forma de abordagem aos RNPB ofertando cuidados médicos específicos com caráter humanizado (BRASIL, 2011).

Um estudo de revisão que avaliou os benefícios do MMC na amamentação apontou que o método melhora a da incidência e duração da amamentação, auxilia positivamente no processo de sucção em recém-nascidos prematuros e atua como um facilitador do aleitamento materno exclusivo (PADILHA; STEIDL; BRAZ, 2014), nesse sentido acrescenta-se o potencial do aleitamento materno exclusivo uma vez que o aleitamento prioriza a vida, reduz a incidência de infecções e promove a proteção necessária para o crescimento e desenvolvimento dos recém-nascidos (COSTA et al., 2013; LAMY, 2008). O aleitamento materno está associado a um melhor desenvolvimento neuropsicomotor com um ano de vida e aumento do QI quando as crianças estiverem com oito anos de vida (TESSIER et al., 2003).

Nesse cenário, destaca-se a importância da atitude proativa dos profissionais de saúde, de informar as mães sobre os benefícios da adoção do MMC para que assim elas possam decidir qual o cuidado é melhor para si e para seu bebê (NEU; ROBINSON; SCHMIEGE, 2013), visto que a qualidade da assistência hospitalar resulta de uma comunicação efetiva entre profissionais de saúde, paciente e seus familiares (VERSIANI et al., 2012). Tendo em vista a importância dos profissionais de saúde para a efetivação da humanização nos serviços de saúde, o Ministério da Saúde do Brasil tem o MMC como uma política direcionada a esses profissionais com o intuito de torná-los agentes do processo de implantação do método, muito embora ainda perceba-se a necessidade da sensibilização dos gestores e a alocação de recursos para a melhoria da infraestrutura dos serviços, o que refletirá na humanização do cuidado de forma mais efetiva (GONTIJO, 2012; VERSIANI et al., 2012), uma vez 
que fornecer um atendimento humanizado nada mais é do que criar um ambiente favorável ao atendimento do paciente por meio de mudanças de comportamento de todos os envolvidos no atendimento (RIBEIRO; SILVEIRA 2015). O MMC é uma forma de atenção que incentiva e valoriza a presença e a participação da mãe e da família na unidade neonatal (BRASIL, 2011). Destaca-se que o MMC é um método de baixo custo que pode ser aplicado a quase todos os RNBP sobre os cuidados da UTI neonatal (FREIRE; GARCIA; LAMY, 2008).

O aumento do vínculo família/mãe e filho promovido pelo MMC leva a melhora da qualidade do desenvolvimento neurocomportamental e psicoafetivo do RNBP, favorece o controle térmico, permite um bom padrão do sono e ciclos de sono-vigília adequados, reduz a ocorrência de choro, favorece o controle eficiente da glicemia capilar, da frequência respiratória e oxigenação, favorece a estimulação sensorial adequada do recémnascido, proporciona o crescimento adequado, reduz o risco de infecção hospitalar e diminui o tempo de internação (CATTANEO et al., 1998; ALI et al., 2009; ACHARYA et al., 2014). A utilização do MMC propicia o fortalecimento do vínculo afetivo, pois beneficia a criação de um clima onde os pais se tornam progressivamente mais conscientes das necessidades de seus filhos RNBP os tornando mais propensos a um cuidado sensível (TESSIER et al., 2003). Os resultados de um estudo realizado na Colômbia fornecem apoio cientifico em relação à segurança da utilização do MMC no cuidado de bebês clinicamente estáveis, uma vez que o método já está incorporado no cuidado de RNBP em muitos hospitais em todo o mundo, nos diferentes níveis de atenção (CHARPAK et al., 1997).

Algumas informações devem ser consideradas na avaliação da confiabilidade e validade desta revisão. Em primeiro lugar a realização da seleção dos artigos por apenas um pesquisador e em segundo lugar somente a utilização de estudos disponíveis publicamente na íntegra.

\section{CONCLUSÃO}

Esta revisão da literatura sugere que o MMC auxilia positivamente no desenvolvimento do RNPB, demonstrando ser uma alternativa de cuidado sem que haja prejuízo algum. Destaca-se que adoção desse método possibilita uma humanização na assistência, estimulo ao vínculo afetivo mãe-filho, melhor a adesão ao aleitamento materno e reduz custo hospitalares. Sendo importante ressaltar que tanto no Brasil, como nos países desenvolvidos, o MMC é uma proposta para beneficiar a saúde do RNBP e não para substituir a tecnologia hoje utilizada nas unidades neonatais.

\section{REFERÊNCIAS}

ACHARYA, N.; SINGH, R. R.; BHATTA, N. K.; POUDEL, P. Randomized Control Trial of Kangaroo Mother Care in Low Birth Weight Babies at a Tertiary Level Hospital. J Nepal Paediatric Society, v. 34, n. 1, p. 18-23, 2014

ALI, S.M.; SHARMA, J.; SHARMA, R.; ALAM, S. Kangaroo Mother Care as compared to conventional care for low birth weight babies. Dicle Tip Dergisi, v. 36, n. 3, p. 155160,2009

ALMEIDA, M. F. D.; ALENCAR, G. P.; SCHOEPS, D.; NOVAES, H. M. D.; CAMPBELL, O.; RODRIGUES, L. C. Sobrevida e fatores de risco para mortalidade neonatal em uma coorte de nascidos vivos de muito baixo peso ao nascer, na Região Sul do Município de São Paulo, Brasil. Cad Saúde Pública, v. 27, n. 6, p. 1088-1098, 2011

BERA, A.; GHOSH, J.; SINGH, A. K.; HAZRA, A.; MUKHERJEE, S.; MUKHERJEE, R. Effect of kangaroo mother care on growth and development of low birthweight babies up to 12 months of age: a controlled clinical trial. Acta Paediatrica, v. 103, n. 6, p. 643-650, 2014

BRASIL. Ministério da Saúde. Secretaria de Atenção à Saúde. Departamento de Ações Programáticas Estratégicas. Atenção humanizada ao recém-nascido de baixo peso: manual técnico. 2. ed. Brasília: Ministério da Saúde, 2011.

BRASIL. Ministério da Saúde. Secretaria de Políticas de Saúde. Área da Saúde da Criança. Atenção humanizada ao recém-nascido de baixo peso: método mãe-canguru: 
manual do curso. Brasília: Ministério da Saúde, 2002.

CARDOSO, A. C. A.; ROMITI, R.; RAMOS, J. L. A.; ISSLER, H.; GRASSIOTTO, C.; SANCHES, M. T. C. Método mãecanguru: aspectos atuais. Pediatria. v. 28, n. 2, p. 128$134,2006$.

CATTANEO, A.; DAVANZO, R.; WORKU, B.; SURJONO, A.; ECHEVERRIA, M.; BEDRI, A.; HAKSARI, E.; OSORNO, L.; GUDETTA, B.; SETYOWIRENI, D.; QUINTERO, S.; TAMBURLINI, G. Kangaroo mother care for low birthweight infants: a randomized controlled trial in different settings. Acta Paediatrica, v. 87, n. 9, p. 976985, 1998.

CHARPAK, N.; RUIZ-PELÁEZ, J.G.; FIGUEROA, Z.; CHARPAK, Y. Kangaroo mother versus traditional care for newborn infants $\leq 2000$ grams: a randomized, controlled trial. Pediatrics, v. 100, n. 4, p. 682-688, 1997.

COSTA, L. K. O.; QUEIROZ, L. L. C.; SILVA QUEIROZ, R. C. C.; RIBEIRO, T. S. F.; FONSECA, M. D. S. S. Importância do aleitamento materno exclusivo: Uma revisão sistemática da literatura. Revista de Ciências da Saúde, v. 15, n. 1, 2013.

FREIRE, N. B. S.; GARCIA, J. B. S.; LAMY, Z. C. Evaluation of analgesic effect of skin-to-skin contact compared to oral glucose in preterm neonates. Pain, v. 139, n. 1, p. 28-33, 2008.

GHAVANE, S.; MURKI, S.; SUBRAMANIAN, S.; GADDAM, P.; KANDRAJU, H.; THUMALLA, S. Kangaroo Mother Care in Kangaroo ward for improving the growth and breastfeeding outcomes when reaching term gestational age in very low birth weight infants. Acta Paediatrica, v. 101, n. 12, p. e545-e549, 2012.

GONTIJO, T. L.; XAVIER, C. C.; FREITAS, M. I. F. Avaliação da implantação do Método Canguru por gestores, profissionais e mães de recém-nascidos. Cad Saúde Pública, v. 28, n. 5, p. 935-944, 2012.

LAMY, F. F.; SILVA, A. A. M.; LAMY, Z. C.; GOMES, M. A. S. M.; MOREIRA, M. E. L. Avaliação dos resultados neonatais do método canguru no Brasil. J Pediatr, v. 84, n. 5, p. 428-435, 2008.

MACHADO, A. C. D. B.; GIRÃO, G. C. L. R.; RODRIGUES, L. C. L.; COSTA, S. S. D. Produção do conhecimento sobre o método canguru. Revista Interdisciplinar NOVAFAPI, v. 5, n. 1, p. 61-67, 2012.

MOTA, L. A.; SÁ, F. E.; FROTA, M. A. Estudo comparativo do desenvolvimento sensório-motor de recém-nascidos prematuros da unidade de terapia intensiva neonatal e do método canguru. Rev Bras Promoc Saude, v. 18, n. 4, p. 191-198, 2005.

NAGAI, S.; YONEMOTO, N.; RABESANDRATANA, N.; ANDRIANARIMANANA, D.; NAKAYAMA, T.; MORI, R. Longterm effects of earlier initiated continuous Kangaroo Mother Care (KMC) for low-birth-weight (LBW) infants in Madagascar. Acta Paediatrica, v. 100, n. 12, p. e241-e247, 2011.

NAGAI, S.; ANDRIANARIMANANA, D.; RABESANDRATANA, N.; YONEMOTO, N.; NAKAYAMA, T.; MORI, R. Earlier versus later continuous Kangaroo Mother Care (KMC) for stable low-birth-weight infants: a randomized controlled trial. Acta Paediatrica, v. 99, n. 6, p. 826-835, 2010

NEU, M.; ROBINSON, J.; SCHMIEGE, S. J. Influence of holding practice on preterm infant development. MCN MCN Am J Matern Child Nurs, v. 38, n. 3, p. 136, 2013

PADILHA, J. F.; STEIDL, E. M. S.; BRAZ, M. M. Efeitos do método mãe-canguru em recém-nascidos pré-termo. Fisioterapia Brasil, v. 15, n. 2, p. 147-153, 2016

PRISMA. Transparent Reporting of Systematic Reviews and Meta-Analyses. Disponível em: <http://www. prisma-statement.org/> . Acesso em: 05 maio 2015.

RIBEIRO, I; SILVEIRA, M. G. C. C. Humanização hospitalar no Sistema Único de Saúde. Rev Interdisciplinar Ciências e Saúde, v. 2, n. 3, p. 19-24, 2015.

SPEHAR, M. C. Mães de bebês prematuros no Método Canguru: aspectos psicossociais, enfrentamento e autoeficácia. 2013. 126f. Dissertação (Mestrado em Processos de Desenvolvimento Humano e Saúde) Instituto de Psicologia da Universidade de Brasília, Universidade de Brasília, Brasília, DF.

SUMAN RAO, P. N.; UDANI, R.; NANAVATI, R. Kangaroo mother care for low birth weight infants: a randomized controlled trial. Indian pediatrics, v. 45, n. 1, p. 17, 2008 
TESSIER, R.; CRISTO, M. B.; VELEZ, S.; GIRON, M.; NADEAU, L.; CALUME, Z. F.; RUIZ-PALÁEZ, J. G.; CHARPAK, N. Kangaroo Mother Care: A method for protecting high-risk low-birth-weight and premature infants against developmental delay. Infant Behavior and Development, v. 26, n. 3, p. 384-397, 2003

VERSIANI, C. C.; SILVA, K. M.; BRETÃS, T. C.; MARQUES, F.; SOUTO, S. G.; MAGALHÃES, D. O. Humanização da assistência de enfermagem nos serviços de urgência e emergência hospitalar: um desafio. EFDeprtes.com/ Revista digital, v. 17, n. 170, 2012. Disponível em: $<$ http://www.efdeportes.com/efd170/humanizacao-daassistencia-de-enfermagem.htm/ $>$. Acesso em: 12 out. 2015.

VICTORA, C. G.; AQUINO, E. M.; DO CARMO LEAL, M.; MONTEIRO, C. A.; BARROS, F. C.; SZWARCWALD, C. L. Saúde no Brasil Dois: Saúde de mães e crianças no Brasil: progressos e desafios. The Lancet, v. 377, n. 9780, p. 1863-1876, 2011.

WHITELAW, A.; HEISTERKAMP, G.; SLEATH, K.; ACOLET, D.; RICHARDS, M. Skin to skin contact for very low birthweight infants and their mothers. Arch Dis Child Educ., v. 63, n. 11, p. 1377-1381, 1988.

WORLD HEALTH ORGANIZATION (WHO). Born too soon: the global action report on preterm birth. Geneva, 2012. Disponível em: <http://www.who.int/maternal_ child_adolescent/documents/born_too_soon/en/ $>$. Acesso em: 18 out. 2015.

Recebido em: 04 de agosto de 2016 Aceito em: 30 de dezembro de 2016 